\title{
Empirical maximum lifespan of earthworms is twice that of mice
}

\author{
Christian Mulder • Rob Baerselman • \\ Leo Posthuma
}

Received: 16 March 2007 / Accepted: 3 July 2007 / Published online: 9 August 2007

(C) American Aging Association 2007

\begin{abstract}
We considered a Gompertzian model for the population dynamics of Eisenia andrei casecohorts in artificial OECD soil under strictly controlled conditions. The earthworm culture was kept between 18 and $22^{\circ} \mathrm{C}$ at a constant $\mathrm{pH}$ of 5.0. In all, 77 lumbricids were carefully followed for almost 9 years, until the oldest died. The Eisenia median longevity is 4.25 years and the oldest specimen was 8.73 years. Eisenia cocoons were hand-sorted every 3 weeks, washed in distilled water, placed in Petri dishes, and counted. Regular removal did not reduce breeding. Each fertile cocoon contained on average two or three embryos. The failure rates (mortality and infertility percentages) are smooth power functions where the rate at time $(n+1)$ captured most of the phenomenology of the previous rate at time $n$, as expected by the considered law, but not at both the beginning and the end of this long-term laboratory study.
\end{abstract}

Keywords Allometric scaling · Body-mass values . Cocoon fertility $\cdot$ Eisenia $\cdot$ Failure rates $\cdot$ Gompertz

Classic allometry postulates that most growth rates of two systems (from organs up to populations) are related by a power function in which the time variable

C. Mulder $(\bowtie) \cdot$ R. Baerselman $\cdot$ L. Posthuma

National Institute for Public Health and the Environment, P.O. Box 1, 3720BA Bilthoven, The Netherlands e-mail: Christian.Mulder@RIVM.nl is not incorporated, in contrast to the age-related kinetics predicted by the almost universal Gompertzian law (Gompertz 1825; Witten 1985). Any association between aging and energy metabolism has several ecological implications (Speakman 2005). Among others, the most relevant implication is that maternal age effects, characterized by a negative relationship between age and fecundity, occur in most taxa regardless of their average body size. In contrast to deuterostomes, whose vouchers or records are often kept by zoological collections, little is known on the complete life-history of protostomes. In view of the relevance of soil invertebrates in current experiments, a case-cohort was built up in a biosafety level-3 facility to allow a continuous monitoring of lumbricids born in captivity. No stress was introduced in our experimental design and the use of a set of $2 \times 4$ replicates avoided any overcrowding.

The median longevity of Eisenia andrei is 63 months $\pm 27.3 \mathrm{SD}$. The median survivorship of $E$. andrei is 240 weeks. Of the 77 Eisenia involved in our study, 8 lived as long as 8 years and 4 adults almost reached a 9-year lifespan (Table 1). Our oldest specimen became 105 months old, an exceptional record for earthworms. In this case-cohort, the observed longevity is directly correlated to the median bodymass of the adults (all the adults: $F$-value $=52.98, p=$ $3 \times 10^{-10}$; adults from large cocoons: mixed $F$-value $=$ 9.15, $p=0.0044$; adults from small cocoons: mixed $F$ value $=8.14, p=0.0072)$. $E$. andrei showed an individ- 
Table 1 Viability of the Eisenia case-cohort. Adults' averages and standard deviation per year; body-mass averages in $\mathrm{mg}$ (fresh weight and standard deviation per year); cocoons determined by hand-sorting every 3 weeks

\begin{tabular}{llll}
\hline Year & Living adults & Fresh body-mass $(\mathrm{mg})$ & Total cocoons \\
\hline 1992 & $76.5 \pm 0.5$ & $671.8 \pm 248.5$ & 5,799 \\
1993 & $73.3 \pm 1.3$ & $876.2 \pm 220.5$ & 6,334 \\
1994 & $71.4 \pm 0.9$ & $832.4 \pm 198.4$ & 4,864 \\
1995 & $57.4 \pm 9.9$ & $781.3 \pm 209.2$ & 3,523 \\
1996 & $41.8 \pm 2.6$ & $959.8 \pm 279.5$ & 4,148 \\
1997 & $35.8 \pm 1.7$ & $1,041.1 \pm 372.2$ & 2,576 \\
1998 & $26.6 \pm 4.1$ & $1,038.5 \pm 512.2$ & 825 \\
1999 & $11.8 \pm 4.0$ & $1,090.7 \pm 556.3$ & 310 \\
2000 & $4.0 \pm 1.6$ & $1,045.4 \pm 569.3$ & 0 \\
\hline
\end{tabular}

ual average cocoon production of $0.8-2.0$ cocoons per week (Table 1). 36,455 juveniles were detected, with an average reproduction rate of 2.33 juveniles per fertile cocoon. During the entire experiment, aberrations of all born juveniles were absent, except for two juveniles with joined bodies and shared organs (Siamese twins). The cocoons were pale with a mucousy, jelly-like consistency at birth. For E. andrei, reproduction rate and fresh biomass remain directly correlated (Fig. 1a), in contrast to reproduction rate and time that remain negatively correlated (Fig. 1b). The time-dependent accumulation curves do not show phenomenological distinct endpoint clusters, although the single adults generated from large cocoons show a slightly higher endpoint than those generated from small cocoons (Fig. 1c).

Tests for relationships between the numerical abundance, the fresh body-mass, and the population biology of lumbricids in microcosms were explicitly sought. Decreasing population growths and numerical abundances (here, 'failure') within eight different replicates belonging to the same Eisenia age-cohort at time $n$ were sufficiently robust to encompass the Gompertzian equation $y_{(n+1)}=\alpha y_{n}^{\beta}$, where the failure rate (mortality and infertility percentages) of one group at any age $(n+1)$ is proportional to the rate of that group when it was younger ( $n$, in this case differing 3 weeks in age) raised to the exponent $\beta$. Of the five Gompertzian patterns determined by $\alpha$ and $\beta$ reported by Bajzer (1999), the slow restricted growth $(\alpha=1.96$ and $\beta=0.7$ ) was recognized during the first 170 weeks. In that period, choosing any rate $y$ at time $(n+1)$, the previous rate $y_{n}$ was found to be very close to the observed rate (mortality rate: $F$-value $=380, p=10^{-30}$;
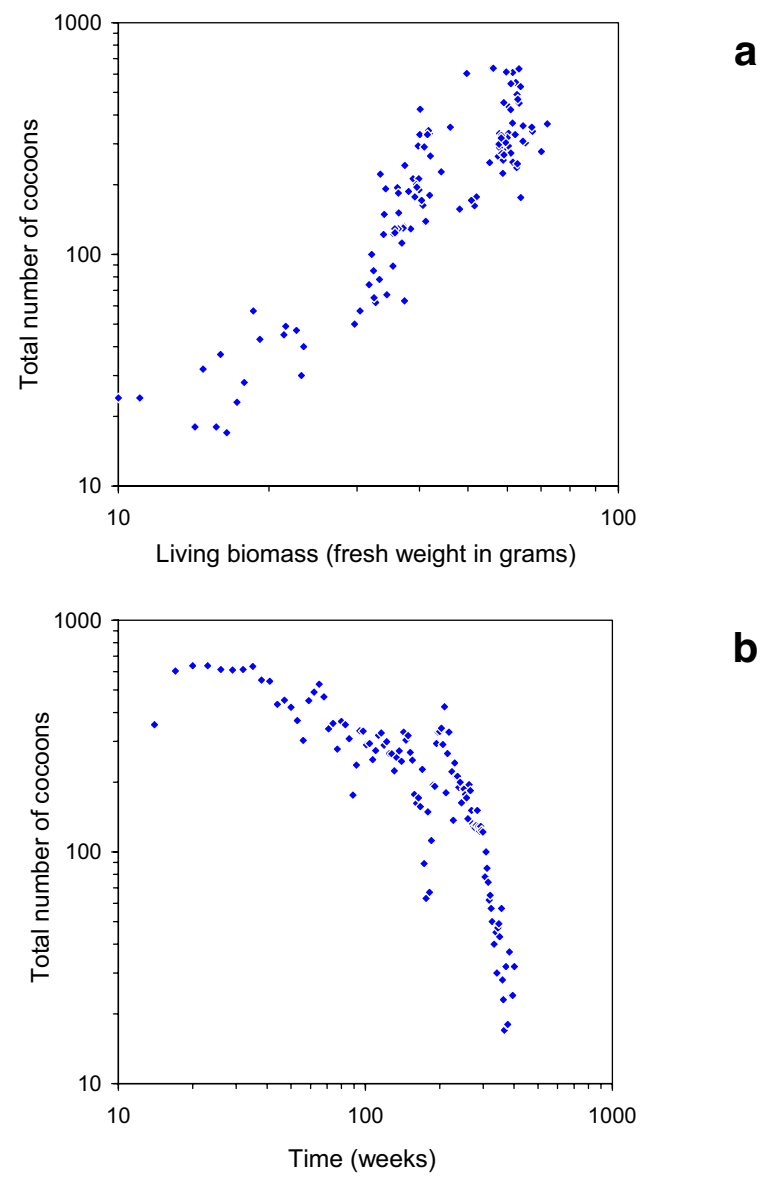

b

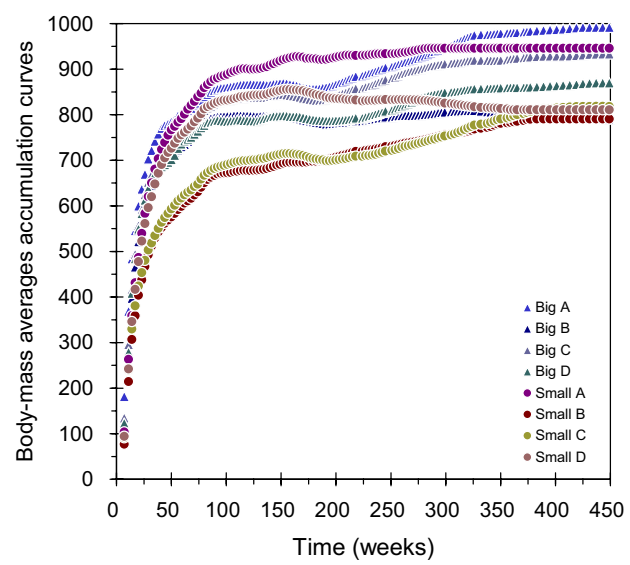

Fig. 1 Total number of cocoons plotted against the biomass of living earthworms and the time of sampling $\left(\log _{10}-\log _{10}\right.$ scatter). a The fresh biomass values of the epigeic Eisenia andrei are directly correlated with the reproduction rate (linear fit: $R^{2}=0.597, F$-value $\left.=173,8 \times 10^{-25}, n=120\right)$. b The reproduction rate is negatively correlated with age (linear fit: $R^{2}=$ $0.76, F$-value $\left.=372, p=4 \times 10^{-38}, n=120\right)$. c Growth curves reflect the former cocoon size class at birth (GLM-MIXED: $p=0.02$ ) 
infertility rate: $F$-value $=366, p=10^{-24}$ ), implying a deterministic dynamics for younger adults. As Bajzer's $\beta$ lies between $2 / 3$ and $3 / 4$, both these scaling exponents of 0.66 and 0.75 were used to compute the Gompertzian failure rates, but the results were not significantly different $(p>0.15)$. Other developmental patterns, like exponential growth, fast restricted growth, and oscillatory growth, were not recognizable. Gompertzian dynamics encompasses Bajzer's growth $(\beta=1)$ by an exponential relationship where $\alpha=\beta=1$. The changing values of the variables $\alpha$ and $\beta$ between week 170 and week 173 reflects the threshold between young ages and middle and old ages. The finding is reasonable (Begun 2006) as it has to do with the failures of the Gompertzian law at both the tails (the early and the late life-stage where aging processes have a finite plateau). Similarly, fresh body-mass values and fertility/productivity rates showed different segments within a same case-cohort, which is not in accordance with any true allometric assumption. Still, a direct relationship between the biomass values of earthworms and those reproduction rates during their entire lifespan can be postulated, as both the resting metabolism and the reproductive senescence of these earthworms seems roughly comparable with other soil invertebrates whose density increases rapidly under favourable conditions (Mulder 2006).

\section{References}

Bajzer Ž (1999) Gompertzian growth as a self-similar and allometric process. Growth Dev Aging 63:3-11

Begun A (2006) Age regularities of the mortality of multicellular organisms. Physica 360A:401-421

Gompertz B (1825) On the nature of the function expressive of the law of human mortality, and on a new mode of determining the value of Life Contingencies. Philos Trans R Soc Lond 115:513-585

Mulder C (2006) Driving forces from soil invertebrates to ecosystem functioning: the allometric perspective. Naturwissenschaften 93:467-479

Speakman JR (2005) Body size, energy metabolism and lifespan. J Exp Biol 208:1717-1730

Witten M (1985) A return to time, cells, systems, and aging: III. Gompertzian models of biological aging and some possible roles for critical elements. Mech Ageing Dev 32:141-177 